\title{
Vogt-Koyanagi-Harada disease - a rare entity in the Polish population - a case report
}

\author{
Martyna Słomska', Julia Kręcicka', Radosław Kaczmarek ${ }^{1,2}$, Anna Turno-Kręcicka ${ }^{1,2}$ \\ 'Department and Clinic of Ophthalmology, University Clinical Hospital in Wroclaw, Poland \\ ${ }^{2}$ Wroclaw Medical University, Poland
}

\section{ABSTRACT}

Vogt-Koyanagi-Harada (VKH) disease is an idiopathic autoimmune reaction against tissues containing melanocytes. Typical eye symptoms are bilateral granulomatous panuveitis and serous retinal detachment (SRD). At a later stage it leads to extensive depigmentation of the fundus ("sunset glow fundus"). The symptoms are accompanied by neurological, auditory or integumentary disorders. The diagnosis is based on clinical findings. In this article we present a case of a 39-year-old man, who had been mistakenly treated for many months before the correct diagnosis was made. Our research is one of the first publications describing VogtKoyanagi-Harada disease in the Polish population; therefore this diagnosis is rarely considered.

KEY WORDS: Vogt-Koyanagi-Harada disease, bilateral granulomatous panuveitis, serous retinal detachment, subretinal fibrosis.

\section{INTRODUCTION}

The name of Vogt-Koyanagi-Harada (VKH) disease derives from the names of three doctors, who independently described this syndrome at the beginning of the $20^{\text {th }}$ century. It occurs mainly in darkly pigmented races, such as Asian, Hispanics, American Indians and Asian Indians. The exact cause of the disease is still unknown, but based on many studies, it is assumed to be an idiopathic T-cell mediated autoimmune process directed against organs containing melanocytes [1], such as eye, hair, inner ear and central nervous system. Many studies on various ethnic groups, including Japanese, Hispanic, Korean, Chinese and others, have documented an association between VKH disease and DR4 haplotype. The highest risk applies to the allele HLA-DRB1 ${ }^{\star} 0405$ [2]. It is still unknown what triggers the immune system; researchers suggest the role of Epstein-Barr virus, cytomegalovirus, interferon therapy in chronic hepatitis $\mathrm{C}$ and skin injury.

\section{CASE REPORT}

The 39-year-old man reported to the nearest ophthalmologist in October 2017, because of blurred vision in the right eye. Visual acuity was 0.2 in the right eye and 1.0 in the left eye. His medical history revealed tinnitus, which was explained by chronic inflammation of the eustachian tube of the left ear and anxiety disorder treated by a psychiatrist. Based on optical coherence tomography (OCT) and fluorescein an- giography (FA) examination, he was diagnosed with central serous retinopathy in the right eye and treated with eplerenone. In March 2018 the patient complained of deteriorated vision in the right eye again; at that time visual acuity was 0.6. Ophthalmologic examination showed subretinal fluid in the macula and serous retinal detachment (SRD) (Figure 1). He was scheduled for phacovitrectomy of the right eye, but the procedure was abandoned due to suspicion of infectious SRD. In April 2018 he was diagnosed with central serous retinopa-

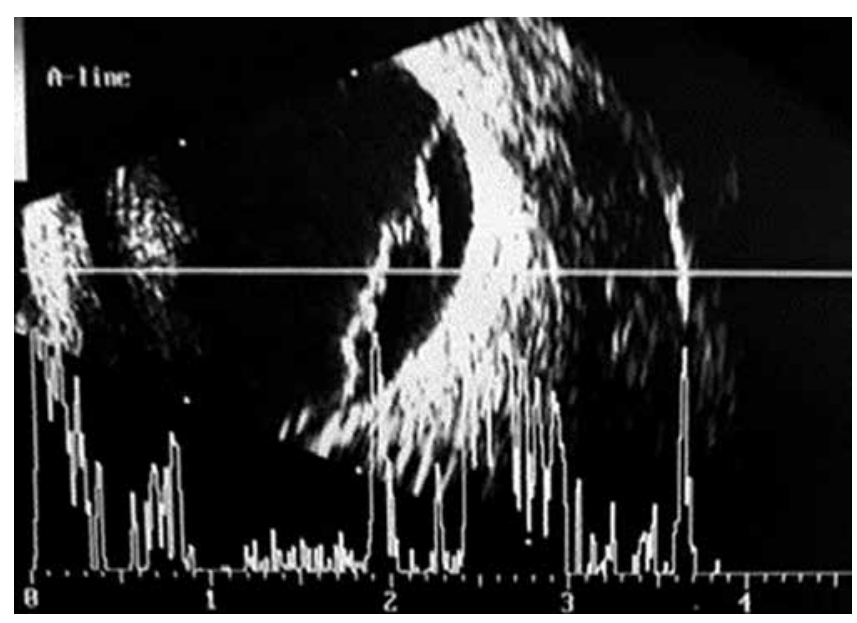

Figure 1. Ultrasonography of the right eye showing serous retinal detachment and thickening of the posterior choroid in March 2018 
thy also in the left eye with visual acuity 0.8 (Figure 2). Based on the presence and high avidity of antibodies to Toxoplasma in laboratory tests and despite the absence of ocular toxoplasmosis symptoms, the patient was treated with cotrimoxazole and prednisolone. Due to lack of improvement, the treatment was changed to pyrimethamine and sulfadoxine. In May 2018 there was progression to bilateral serous retinal detachment and multiple central serous retinopathy documented in FA (Figure 3). Visual acuity was 0.2 in the right eye and 0.7 in the left eye. The patient was referred to the Department of Ophthalmology at University Hospital in Wroclaw, where the suspicion of VKH disease was raised. Fundus examination revealed choroidal depigmentation and SRD in both eyes (Figure 4). We conducted additional tests such as cerebrospinal fluid (CSF) examination, QuantiFERON, angiotensinconverting-enzyme (ACE), anti-HCV and anti-HIV antibodies, HLA DRB1, X-ray and computed tomography (CT) of the chest and magnetic resonance imaging (MRI) of the brain. HLA DRB3, HLA DRB4 genetic testing and QuantiFERON were positive, while other results were within the normal range. In the early stage of $\mathrm{VKH}$ disease, we can often observe pleocytosis in the CSF, which did not occur in our case, probably because the examination was performed a few months after the onset of the first symptoms.

The following treatment was given: methylprednisolone $1 \mathrm{~g} /$ day for three days i.v., next prednisolone $75 \mathrm{mg} /$ day, methotrexate $25 \mathrm{mg}$ per week and acetazolamide $125 \mathrm{mg}$ three times per day. Due to positive QuantiFERON, after pulmonological consultation, rifampicin and nidrazide were added. During treatment, we observed resorption of subretinal fluid. However, due to months of untreated inflammation, the typical VKH disease complication appeared - subretinal fibrosis in the macula of both eyes (Figure 5). Visual acuity decreased to 0.15 in the right eye and counting fingers from $1 \mathrm{~m}$ in the left eye. In July 2018 we decided to perform 23 gauge vitrectomy with removal of fibrous membranes from the subretinal space, endolaser coagulation, retinotomy and

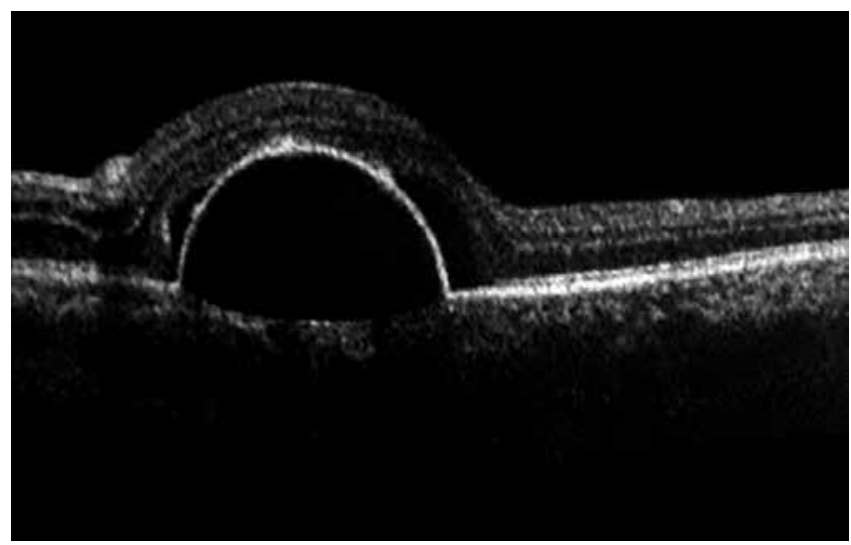

Figure 2. Central serous retinopathy in the left eye in April 2018

silicone oil tamponade in the left eye. Three months later the same operation was performed in the right eye (Figure 6). The patient's best-corrected visual acuity (BCVA) in October 2018 , after this surgery, was 0.1 in the right eye and 0.3 in the left eye.

The patient remained under our observation for the next seven months. During this time, the prednisolone dose was slowly reduced (to $30 \mathrm{mg}$ during the last control) while maintaining methotrexate and adding adalimumab. We did not observe a recurrence of uveitis or choroidal neovascularization (CNV). However, in November 2018, macular edema secondary to subretinal fibrosis appeared in the right eye and two months later also in the left eye (Figure 7). We introduced periocular injections of triamcinolone, which resulted in reduction of macular fluid in the left eye, but there was no reaction in the right eye. Moreover, another complication occurred: subcapsular cataract in both eyes. The patient underwent phacoemulsification with an intraocular lens implant in the left eye. During the last visit in May 2019 the patient's BCVA was counting fingers from $1 \mathrm{~m}$ in the right eye and 0.08 in the left eye. Then, due to the long distance to our clinic, he decided to continue treatment
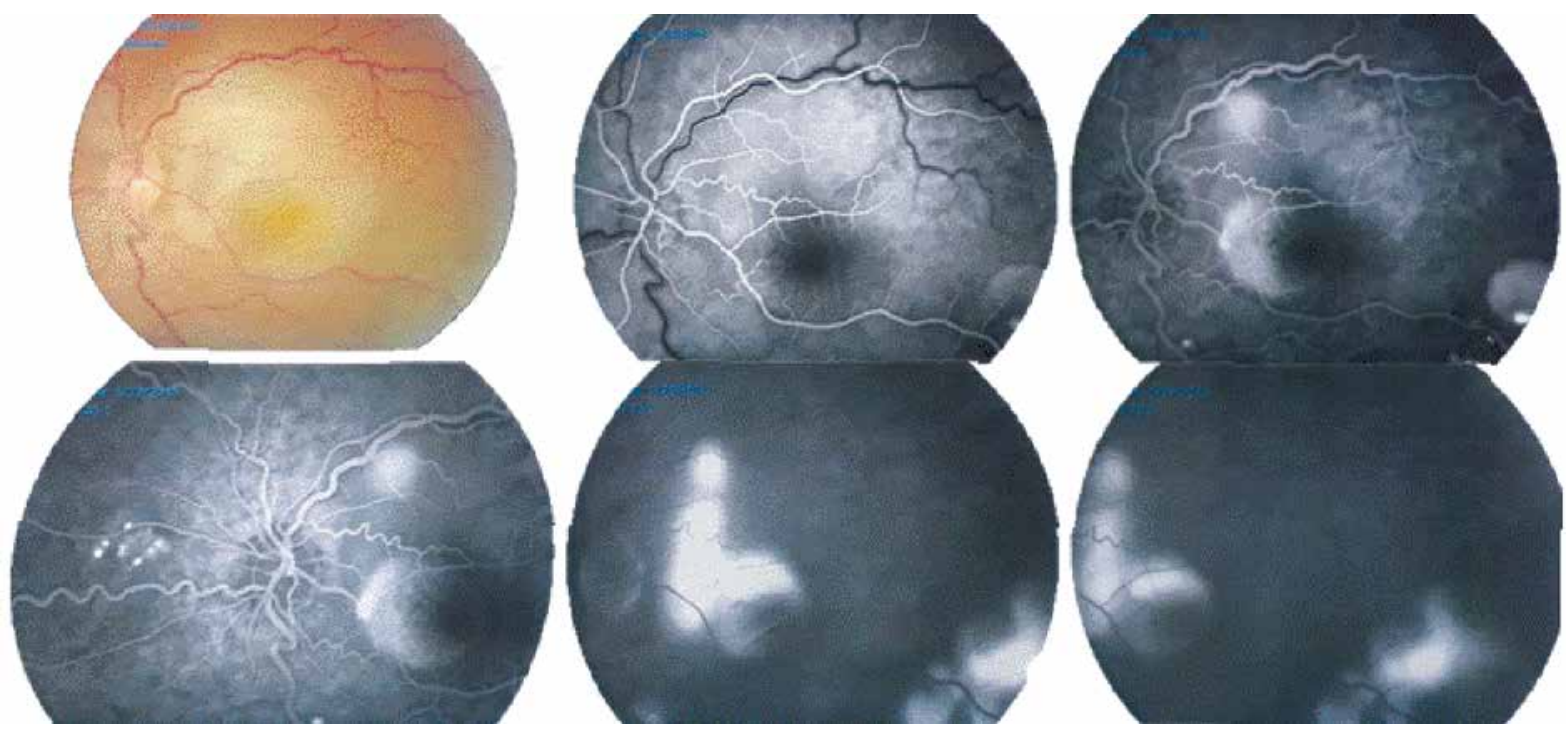

Figure 3. Fluorescein angiography showing multiple central serous retinopathy and serous retinal detachment in the left eye in May 2018 

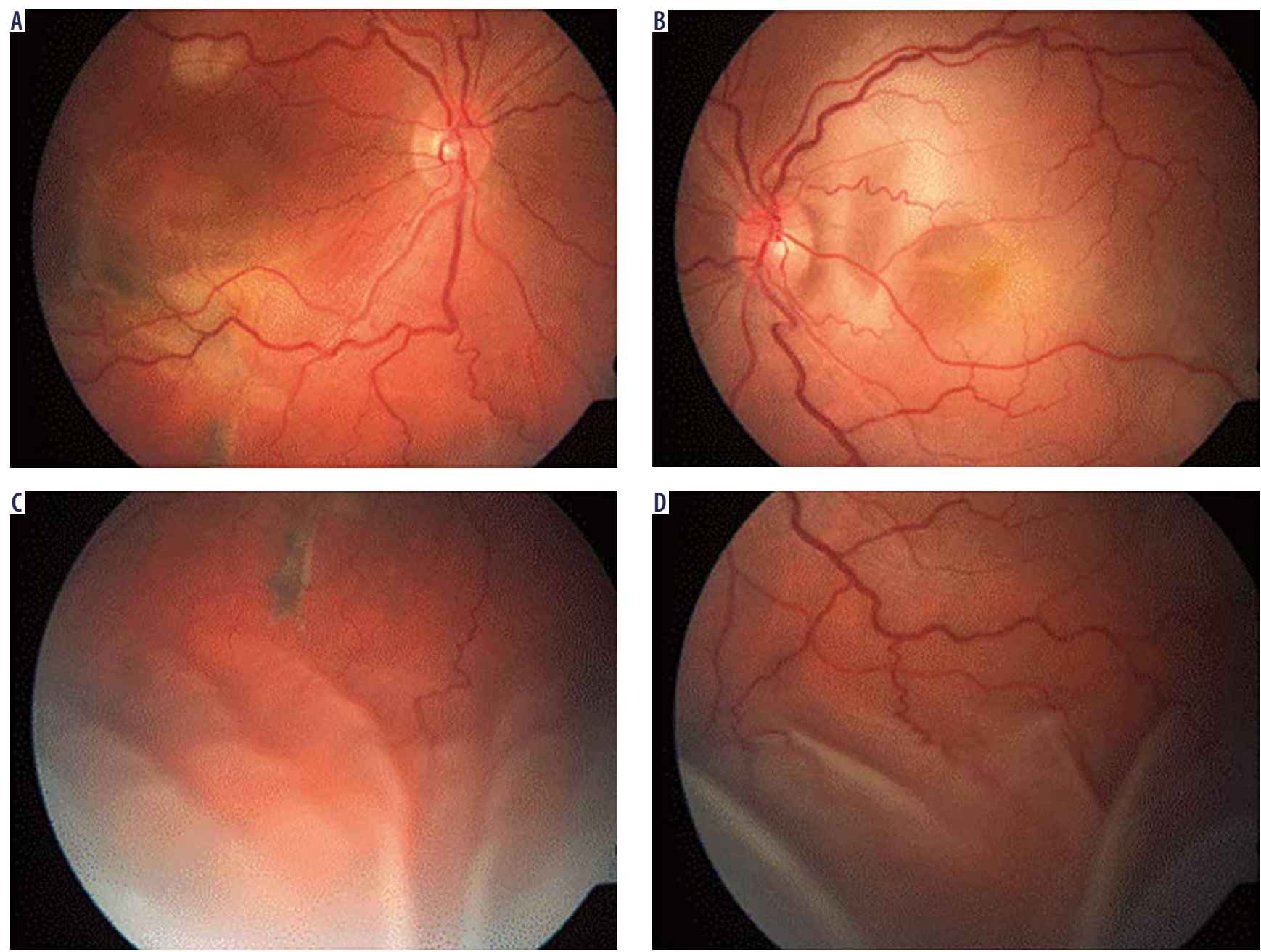

Figure 4. Choroidal depigmentation and serous retinal detachment in both eyes in May 2018

closer to his area, where the therapy was extended with antiVEGF injections.

\section{DISCUSSION}

The diagnosis of $\mathrm{VKH}$ disease is mainly based on clinical examination. Read et al. established Revised Diagnostic Crite-

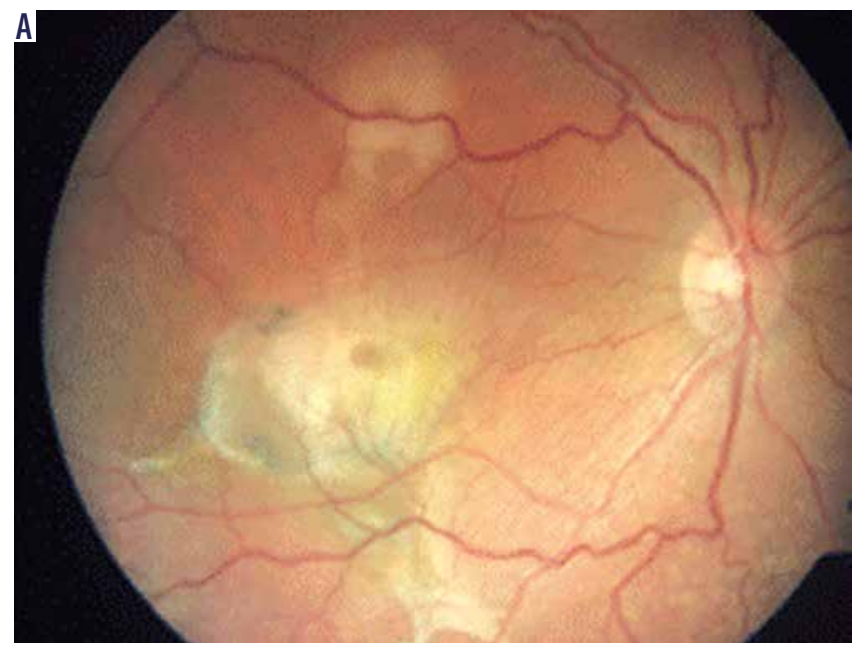

Figure 5. Subretinal fibrosis in the macula in July 2018 ria for VKH disease during the First International Workshop on Vogt-Koyanagi-Harada disease in 1999. They are presented in Table I [3]. Based on the findings in our patient we can diagnose incomplete VKH disease, as he meets 1-4 criteria.

To support the diagnosis, ancillary tests should be done. One of the most useful is fluorescein angiography. It reveals

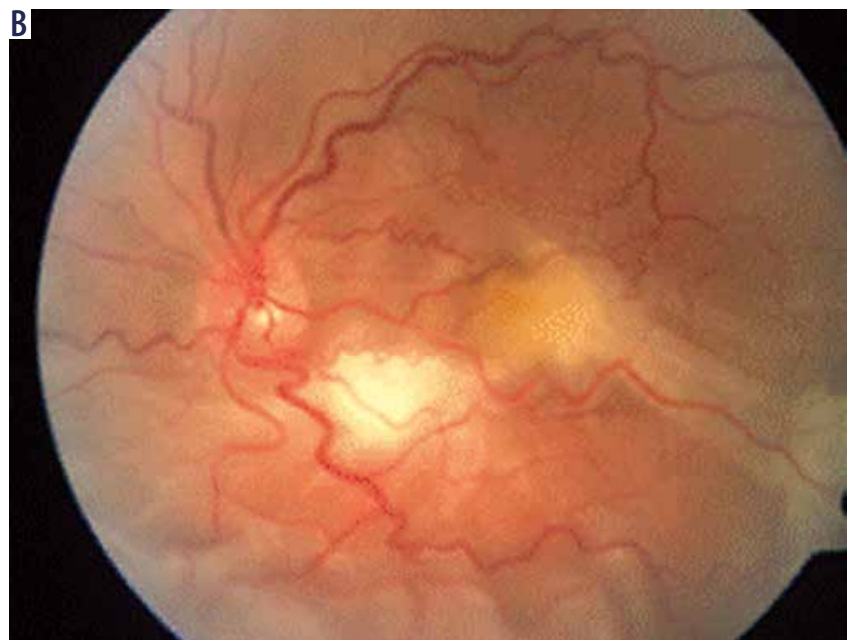



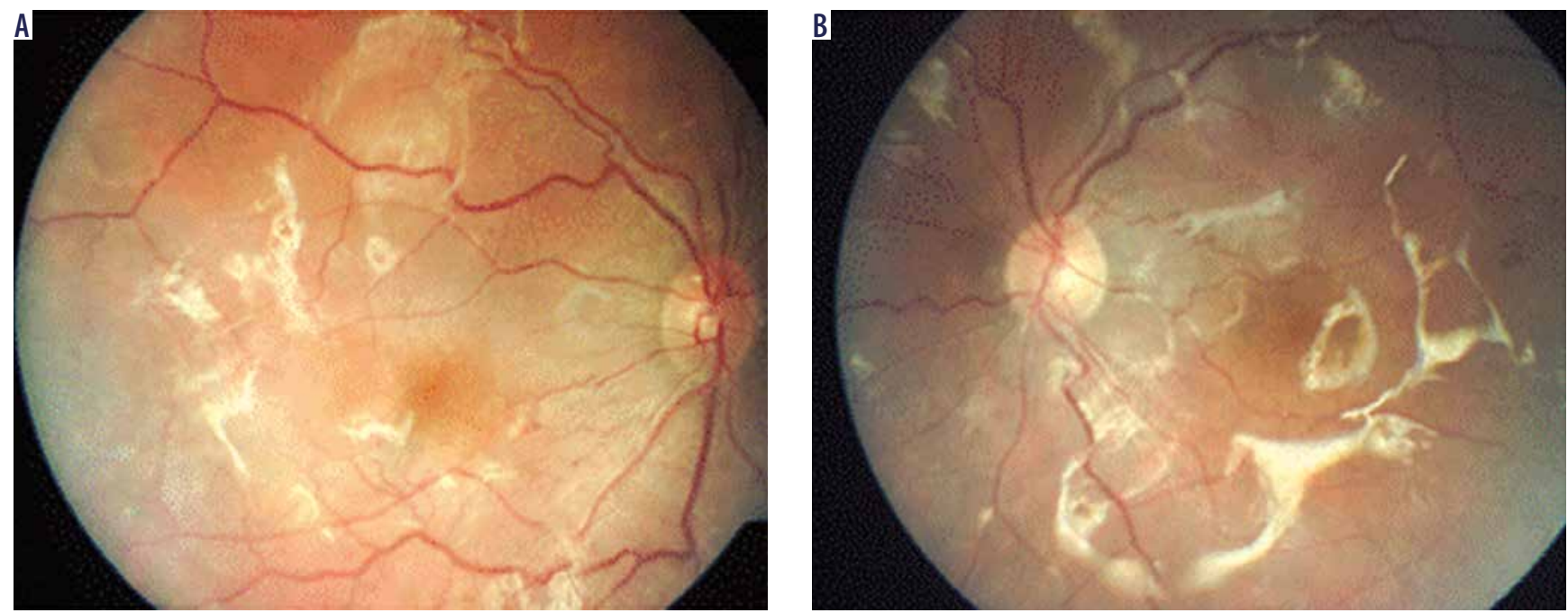

Figure 6. Fundus examination after vitrectomy
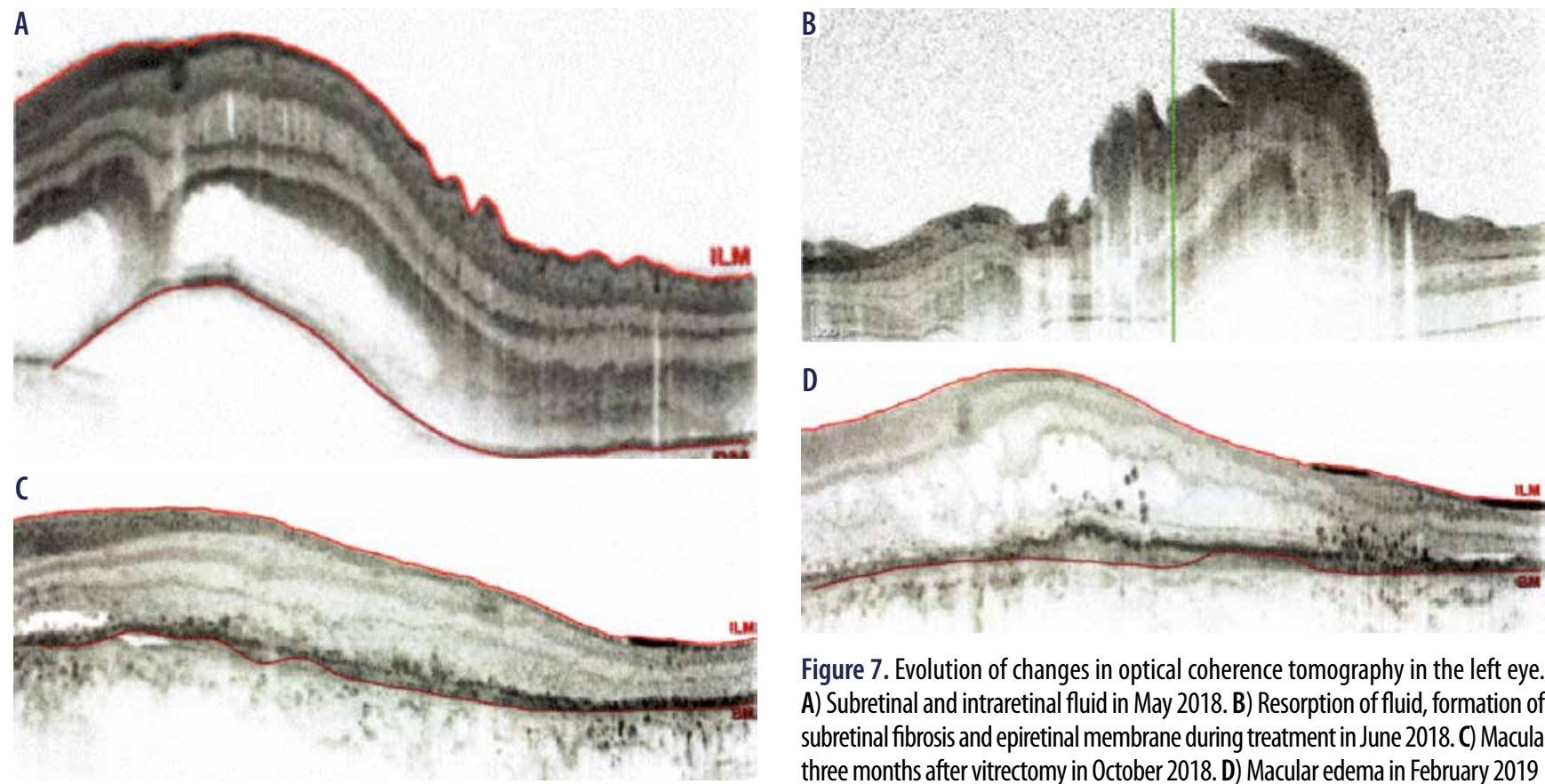

Figure 7. Evolution of changes in optical coherence tomography in the left eye. A) Subretinal and intraretinal fluid in May 2018. B) Resorption of fluid, formation of subretinal fibrosis and epiretinal membrane during treatment in June 2018. C) Macula three months after vitrectomy in October 2018. D) Macular edema in February 2019

numerous hyperfluorescent spots at the level of the retinal pigment epithelium (RPE), which increase over time and fill the subretinal space, forming serous retinal detachment. The optic disc also can show leakage. Moreover, FA can demonstrate delay in choroidal perfusion and complications such as $\mathrm{CNV}$ [1]. Ultrasonography can reveal thickening of the posterior choroid or sclera, SRD and vitreous opacities [4]. To monitor disease activity and evaluate treatment, the noninvasive examination OCT is used. It can show subretinal fluid with subretinal septa, which divide this space into several compartments. It can also reveal intraretinal edema, undulation of RPE, ellipsoid zone damage, choroidal thickness, choroidal folds or choroidal hyperreflective dots in the acute stage [5]. Another performed test is fundus autofluorescence (FAF). In the acute stage, we can observe hyperautofluores-

cence in the macula with hypoautofluorescence in the areas of the serous retinal detachment. However, in the chronic stage, different patterns may be present, such as increased, decreased or normal autofluorescence [5].

It is very important to start treatment quickly and aggressively to suppress intraocular inflammation and prevent complications. The basis of the therapy is high-dose oral corticosteroids, usually in the range of $1-1.5 \mathrm{mg} / \mathrm{kg} /$ day with slow reduction within 6 months or longer. Therapy may be preceded by intravenous application of methylprednisolone $1 \mathrm{~g} /$ day for three consecutive days [6]. Patients with resistant or recurrent disease or who cannot tolerate steroids should be treated with immunosuppressive therapy (IST), such as cyclosporine, methotrexate, mycophenolate mofetil, azathioprine or others. Some authors even suggest IST as first-line therapy 
Table I. Diagnostic criteria for Vogt-Koyanagi-Harada disease [3] Complete Vogt-Koyanagi-Harada disease (criteria 1 to 5 must be present)
1. No history of penetrating ocular trauma or surgery preceding the initial onset of uveitis
2. No clinical or laboratory evidence suggestive of other ocular disease entities
3. Bilateral ocular involvement ( $a$ or $b$ must be met, depending on the stage of disease when the patient is examined)
a. Early manifestations of disease
(1) There must be evidence of a diffuse choroiditis (with or without anterior uveitis, vitreous inflammatory reaction, or optic disc hyperemia), which may manifest as one of the following:
(a) Focal areas of subretinal fluid, or
(b) Bullous serous retinal detachments
(2) With equivocal fundus findings; both of the following must be present as well:
(a) Focal areas of delay in choroidal perfusion, multifocal areas of pinpoint leakage, large placoid areas of hyperfluorescence, pooling within subretinal fluid, and optic nerve staining (listed in order of sequential appearance) by fluorescein angiography, and
(b) Diffuse choroidal thickening, without evidence of posterior scleritis on ultrasonography

b. Late manifestations of disease

(1) History suggestive of prior presence of findings from 3a, and either both (2) and (3) below, or multiple signs from (3):

(2) Ocular depigmentation (either of the following manifestations is sufficient):

(a) Sunset glow fundus, or

(b) Sugiura sign

(3) Other ocular signs:

(a) Nummular chorioretinal depigmented scars, or

(b) Retinal pigment epithelium clumping and/or migration, or

(c) Recurrent or chronic anterior uveitis

4. Neurological/auditory findings (may have resolved by time of examination)

a. Meningismus (malaise, fever, headache, nausea, abdominal pain, stiffness of the neck and back, or a combination of these factors; headache alone is not sufficient to meet definition of meningismus, however), or

b. Tinnitus, or

c. Cerebrospinal fluid pleocytosis

5. Integumentary finding (not preceding onset of central nervous system or ocular disease)

a. Alopecia, or

b. Poliosis, or

c. Vitiligo

Incomplete Vogt-Koyanagi-Harada disease (criteria 1 to 3 and either 4 or 5 must be present)

Probable Vogt-Koyanagi-Harada disease (isolated ocular disease; criteria 1 to 3 must be present)

Authors' footnote: Sunset glow fundus - pale choroidal pigmentation, Sugiura sign - perilimbal vitiligo.

to avoid the numerous side effects associated with long-term corticosteroid treatment [7]. Therapy may be successfully supported by intravitreal injections of anti-VEGF for the treatment of CNV [8] or triamcinolone acetonide for control of inflammation during the acute stage. In associated anterior segment uveitis topical corticosteroids are recommended. To reduce ciliary spasm and prevent creation of posterior synechia cycloplegic-mydriatic eye drops should be added [9].

If the proper treatment is started rapidly and aggressively, the prognosis is quite good, with almost two thirds of patients retaining BCVA 0.5 or better [10]. The prognosis is worsened by occurrence of many complications, long duration of the disease, worse acuity at presentation, older age at the onset of disease, and frequent recurrent episodes [11].

The most common complications are cataract, glaucoma, CNV and subretinal fibrosis (SRF). The last two are the main reason for the worse final visual acuity, especially when they involve the fovea. In 2018 Ahao et al. published a pioneer study about surgical treatment of SRF in VKH disease. They presented the results including 7 eyes of 6 patients, who underwent vitrectomy with retinotomy and SRF removal, followed by tamponade of gas or silicone oil. Six of 7 eyes showed improved visual acuity after surgery and 4 of them even achieved BCVA 0.1 or better. In one case, where 
the SRF strongly attached around the optic disc, surgery was complicated by massive subretinal hemorrhage resulting in the loss of light perception [12]. Subretinal fibrosis also developed in our patient's macula in both eyes, including the entire fovea in the left eye. Attempting to improve visual acuity, we performed vitrectomy with removal of fibrous membranes, achieving improved BCVA in the left eye from counting fingers from $1 \mathrm{~m}$ to 0.3 , while BCVA in the right eye remained similar.

\section{CONCLUSIONS}

Vogt-Koyanagi-Harada disease is very rare in Central Europe, but should be considered in patients presenting with bilateral granulomatous panuveitis and serous retinal detachment, because the prognosis depends on early diagnosis and appropriate anti-inflammatory treatment.

\section{DISCLOSURE}

The authors declare no conflict of interest.

\section{References}

1. Attai S, Khochtali S, Kahloun R, et al. Vogt-Koyanagi-Harada disease. Expert Rev Ophthalmol 2012; 7: 565-585.

2. Shindo Y, Inoko H, Yamamoto T, et al. HLA-DRB1 typing of Vogt-Koyanagi-Harada's disease by PCR-RFLP and the strong association with DRB1*0405 and DRB1*0410. Br J Ophthalmol 1994; 78: 223-226.

3. Read RW, Holland GN, Rao NA, et al. Revised Diagnostic Criteria for Vogt-Koyanagi-Harada Disease: Report of an International Committee on Nomenclature. Am J Ophthalmol 2001; 131: 647-652.

4. Forster DJ, Cano MR, Green RL, et al. Echographic features of the Vogt-Koyanagi-Harada syndrome. Arch Ophthalmol 1990; 108: 1421-1426.

5. Baltmr A, Lightman S, Tomkins-Netzer 0. Vogt-Koyanagi-Harada syndrome - current perspectives. Clin Ophthalmol 2016; 10: 2345-2361.

6. Lavezzo MM, Sakata VM, Morita C, et al. Vogt-Koyanagi-Harada disease: review of a rare autoimmune disease targeting antigens of melanocytes. Orphanet J Rare Dis 2016; 11: 29.

7. Paredes I, Ahmed M, Foster C. Immunomodulatory therapy for Vogt-Koyanagi-Harada patients as first-line therapy. Ocul Immunol Inflamm 2006; 14: 87-90.

8. Wu L, Evans T, Saravia M, et al. Intravitreal bevacizumab for choroidal neovascularization secondary to Vogt-Koyanagi-Harada syndrome. Jpn J Ophthalmol 2009; 53: 57-60.

9. 0'Keefe GD, Rao NA. Major review: Vogt-Koyanagi-Harada disease. Surv Ophthalmol 2017; 62: 1-25.

10. Moorthy RS, Inomata H, Rao NA. Vogt-Koyanagi-Harada Syndrome. Surv Ophthalmol 1995; 39: 265-292.

11. Read RW, Rechodouni A, Butani N. Complications and prognostic factors in Vogt-Koyanagi-Harada disease. Am J Ophthalmol 2001; 131: 599-606.

12. Zhao C, Zhang M, Gao F, et al. Surgical treatment of subretinal fibrosis caused macular detachment in Vogt-Koyanagi-Harada disease: a pioneer study. Ocul Immunol Inflamm 2018; 26: 154-159. 